Forschungszentrum Karlsruhe

Technik und Umwelt

Wissenschaftliche Berichte

FZKA 5585

\title{
Water Target at the ISIS Spallation Source: Neutrino and Neutron Fluxes
}

R. L. Burman, L. L. Daemen Institut für Kernphysik 



\title{
Forschungszentrum Karlsruhe Technik und Umwelt
}

\author{
Wissenschaftliche Berichte \\ FZKA 5585
}

\section{Water Target at the ISIS Spallation Source: Neutrino and Neutron Fluxes}

\author{
R. L. Burman ${ }^{*}$ and L. L. Daemen ${ }^{1}$
}

Institut für Kernphysik

1 Los Alamos National Laboratory, Los Alamos, New Mexico 87545, USA.

* Permanent address: P-Division, Los Alamos National Laboratory, Los Alamos, New Mexico 87545, USA.

Forschungszentrum Karlsruhe GmbH, Karlsruhe 
Als Manuskript gedruckt

Für diesen Bericht behalten wir uns alle Rechte vor

Forschungszentrum Karlsruhe $\mathrm{GmbH}$

Postfach 3640, $76021 \mathrm{Kar} / \mathrm{sruhe}$

ISSN 0947-8620 


\section{Abstract}

Effects arising from a water target placed in the ISIS spallation facility, used for both neutron and neutrino experiments, are considered. Calculations are presented for the neutrino flux from stopped $\mu^{+}$decay, and for the neutron fluxes from the ISIS moderators, for several versions of a water target situated upstream of the ISIS spallation source. Two possible configurations, that enhance the neutrino flux by $39-80 \%$ and produce relatively little reduction in neutron flux, are outlined. 


\section{Wasser-Target an der ISIS Spallationsquelle: Fluß von Neutronen und Neutrinos}

\section{Zusammenfassung}

Die Auswirkungen eines Wasser-Targets werden dargelegt, welches in die ISIS Spallationsquelle eingebaut wird, die für Experimente mit Neutronen und Neutrinos benutzt wird. Berechnet wird der Fluß von Neutrinos aus dem $\mu^{+}$Zerfall in Ruhe, und von Neutronen aus den ISIS Moderatoren, für unterschiedliche Konfigurationen eines Wasser-Targets, das vor dem ISIS Spallationstarget plaziert wird. Zwei mögliche Konfigurationen, die den Neutrinofluß um 39-80\% erhöhen und den Neutronenfluß relativ wenig verringern, werden herausgestellt. 


\section{Introduction}

The spallation facility ISIS at the Rutherford Appleton Laboratory in Oxfordshire, UK, provides high-intensity pulsed neutron beams for a wide variety of cold to epithermal neutron experiments. The neutrons are produced by interactions of an $800 \mathrm{MeV}$ proton beam with a tantalum or uranium target. In addition, the facility serves as a highintensity pulsed neutrino source for the KARMEN neutrino experiment, operated by a collaboration of the Forschungszentrum Karlsruhe, University of Karlsruhe, University of Bonn, University Erlangen-Nürnberg, Rutherford Appleton Laboratory, Queen Mary and Westfield College, and Oxford University.

The KARMEN experiment[1] takes advantage of the time structure in the ISIS proton beam in the pursuit of a number of nuclear and particle physics studies. The experiment has been designed around a neutrino source[2] based upon $\pi^{+}$and $\mu^{+}$decay at rest. The pions and muons, and the subsequent neutrinos, are produced in the ISIS spallation target: the $\nu_{\mu}$ from $\pi^{+}$decay has an energy of $29.8 \mathrm{MeV}$, the $\nu_{e}$ and $\bar{\nu}_{\mu}$ from $\mu^{+}$decay have the $0-53 \mathrm{MeV}$ Michel spectral shapes. In order to minimize fast neutron background, the KARMEN detector is sited at about $90^{\circ}$ to the proton beam direction, as the fast neutron flux from the spallation target is sharply peaked forward, while the neutrino flux from decay at rest is isotropic.

Because the neutrino cross sections of interest are around $10^{-40} \mathrm{~cm}^{2}$, the neutrino target/detector assemblies are large and the typical experiment of long duration. Even a modest improvement in the neutrino flux can greatly improve the quality of an experiment. This report considers the implementation of a water target, placed in front of the ISIS spallation target, that affords up to an $80 \%$ increase in the neutrino flux. The neutrino Monte Carlo code is briefly described, and calculations of the neutrino fluxes from the present tantalum and uranium ISIS targets, are given in Sec. 2. Calculations of neutrino fluxes with the addition of a water target are given in Sec. 3. In Sec. 4, we give neutron fluxes entering the separate ISIS beam lines for different configurations of water and spallation targets. In Sec. 5 we compare the effects of water and spallation target choice on the neutron fluxes, the neutrino fluxes, and the neutrino backgrounds. Two suggested compromises for enhancing the neutrino fluxes are emphasized in the concluding section.

\section{Neutrino fluxes for the present ISIS target}

Neutrino fluxes from $\pi^{+}$decay at rest were calculated with a Monte Carlo program KARMEN that was designed for spallation targets and beam stop facilities at mediumenergy proton accelerators. A detailed description of the code is available in ref. [3] and so only a brief outline is given here. The program uses proton reaction cross sections, pion production and absorption cross sections, and particle transport to calculate the neutrino fluxes from the decays of positive and negative muons. The proton beam is transported, with energy loss, through the target geometry. At a Monte Carlo chosen 
proton interaction point, positive and negative pions, weighted by the production cross sections, are selected with initial energy and angle according to measured cross sections. As the pions are tracked through the target geometry they are allowed to inelastically scatter, to multiple-Coulomb-scatter, to be absorbed, or to decay.

Absolute normalization was provided by measurements[4] made on an instrumented mockup of a simplified beam stop; the event-by-event production of pions, followed by signals from pion and muon decay, was used to infer the rate of stopped $\pi^{+}$production per incident proton. Data on the stopped $\pi^{+}$rate were taken for mock beam stops composed of copper, water + copper, and lead for proton energies of 716, 766 and 797 $\mathrm{MeV}$. The code was then normalized, by $10 \%$ increases in the overall proton reaction and pion production cross sections, to give the measured stopped $\pi^{+}$rate. It should be noted that these effects changed the pion production spatial distribution but essentially canceled in the pion production rate.

Table 1: Calculated neutrino fluxes with the present tantalum and uranium targets for proton beam energies of 800 and $750 \mathrm{MeV}$. Column 3 gives the neutrino flux from $\mu^{+}$decay at rest in units of $\nu$ per proton, while column 4 gives the $\bar{\nu}_{e}$ background from $\mu^{-}$decay at rest as a fraction of the number in column 3.

\begin{tabular}{lccc}
\hline $\begin{array}{l}\text { Spallation } \\
\text { Target }\end{array}$ & Proton Energy & $\begin{array}{c}\text { Neutrino Flux } \\
\nu_{\mu}, \nu_{e}, \bar{\nu}_{\mu} \\
\left(\nu \mathrm{p}^{-1}\right)\end{array}$ & $\begin{array}{c}\text { Ratio } \\
\bar{\nu}_{e} / \nu_{e} \\
\left(10^{-3}\right)\end{array}$ \\
\hline Tantalum & $(\mathrm{MeV})$ & 0.0448 & 0.62 \\
Uranium & 800 & 0.0401 & 0.72 \\
Tantalum & 750 & 0.0382 & 0.57 \\
Uranium & 750 & 0.0345 & 0.67 \\
\hline
\end{tabular}

The Monte Carlo code KARMEN was then used to calculate the neutrino fluxes at the ISIS spallation source. As input to the KARMEN neutrino code, the ISIS target was modelled[5] with the simplified geometry shown in Fig. 1. The spallation target basically consists of disks, either of tantalum or depleted uranium clad in zircalloy, encased in rectangular steel blocks as shown in Fig. 1a; the spaces between and surrounding the disks are filled with rapidly flowing heavy water as a coolant. Pion production and decay at rest, and hence neutrino production, is concentrated in this heavy-metal target structure which is shown in more detail in Fig. $1 \mathrm{~b}$.

Results of the calculations, for the two target materials (tantalum and uranium) and for the two proton energies ( 750 and $800 \mathrm{MeV}$ ) that have characterized the Rutherford accelerator operation, are given in Table 1 . It is seen that in their present design, the uranium target produces a neutrino flux about $90 \%$ of that from the tantalum target.

Approximately $99 \%$ of the decay $\pi^{+}$do so at rest, and with the subsequent $\mu^{+}$decay produce the $\nu_{\mu}, \nu_{e}$, and $\bar{\nu}_{\mu}$ neutrino beams. The presence of $\bar{\nu}_{e}$ neutrinos would be 
(a)

(b)
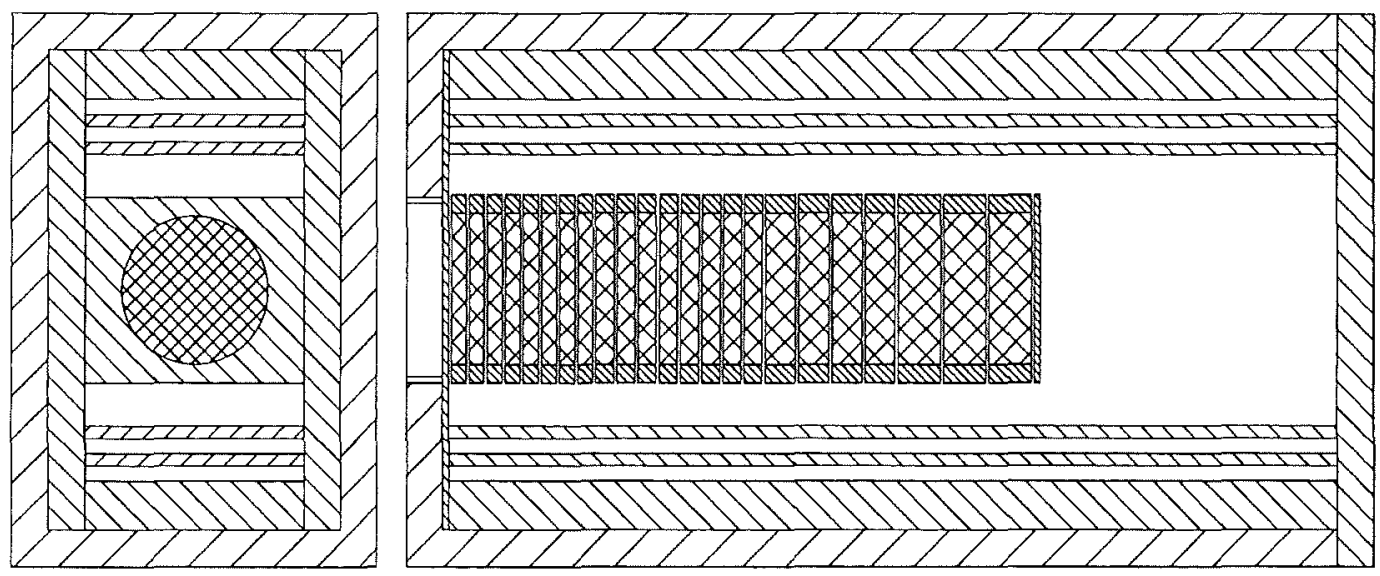

Figure 1: Computer model of the ISIS spallation target, with the heavy-metal target shown in cross-hatching. (a) Frontview of the uranium or tantalum plate structure; (b) topview of the plate structure within the pressure vessel, with the proton beam direction from the left.

a serious background to searches for neutrino oscillations or for rare pion and muon decays. Virtually all $\pi^{-}$that stop are absorbed, and so the possible $\bar{\nu}_{e}$ backgrounds are created from the approximately $1 \%$ of the $\pi^{-}$that decay in flight. The $\mu^{-}$from the decay in flight are tracked until they stop in some particular material; the fraction that leads to decay at rest rather than to absorbtion is calculated and is used to predict the $\bar{\nu}_{e}$ background. These fluxes, as a ratio to the $\nu_{e}$ flux, are shown in the last column of Table 1.

Estimates of the flux uncertainties for the KARMEN decay-at-rest neutrino source are based upon the detailed error analysis for the decay-at-rest fluxes as given in ref[3]. There, as discussed earlier, the absolute normalization of the code was provided by a mock-beamstop experiment[4], LAMPF experiment E866. The measured pion production cross sections used in the KARMEN Monte Carlo code typically have $9.5 \%$ absolute normalization errors. However, because the KARMEN code was instead normalized to the E866 data on stopped $\pi^{+}$per incident proton, this $9.5 \%$ uncertainty is irrelevant and is not included. Error estimates for the present KARMEN experiment are given in Table 2. The main difference between the $\pi^{+}$decay-at-rest and the $\mu^{-}$decay-at-rest uncertainties are in the absolute normalization. A normalization uncertainty for $\pi^{-}$decay in flight is composed of both a $9.5 \%$ contribution from the absolute normalization errors quoted in the pion production measurements, and a $5.0 \%$ contribution from the E866 experiment. This latter number comes from the part of the "systematic effects in E866" entry in Table 2 that represents a possible change in the code normalization.

In addition, a larger number $(5.0 \%)$ is used for the $\pi^{-}$decay-in-flight flux simulation error. The number of pions that can decay in flight are quite sensitive to the open 
Table 2: Estimated errors in the calculated neutrino fluxes from $\pi^{+}$decay at rest and $\mu^{-}$decay at rest.

\begin{tabular}{lcc}
\hline Source of uncertainty & $\begin{array}{c}\pi^{+} \text {Decay at rest } \\
(\%)\end{array}$ & $\begin{array}{c}\mu^{-} \\
\text {Decay at rest } \\
(\%)\end{array}$ \\
\hline Fit of E866 data & 2.4 & - \\
Cross section error & - & 9.5 \\
Systematic effects in E866 & 5.9 & 5.0 \\
ISIS simulation & 0.4 & 5.0 \\
Proton beam energy & 0.3 & 0.3 \\
Protons on target & 2.0 & 2.0 \\
Proton geometry & 0.5 & 0.5 \\
Quadrature sum & & \\
\hline
\end{tabular}

spaces between spallation target components. The elements of the target in-line with the proton beam are quite well defined, but the moderator and shielding surrounding the target are not so well described. Therefore, computer runs were made with movements of various of the shielding components. Typically, for a movement of $2.5 \mathrm{~cm}$, the decayin-flight flux changed up to $8 \%$. A reasonable estimate of the flux uncertainty from the open space uncertainty is $4.5 \%$; this, folded with the $2.0 \%$ uncertainty from component simulation, is the entry in Table 2.

\section{Neutrino fluxes for added heavy-water targets}

Early considerations in the design of a neutrino facility for the beam stop of the Los Alamos Meson Physics Facility (LAMPF) had led to the installation of a $20 \mathrm{~cm}$ water target before the copper beam stop. Because the $\mathrm{A}\left(\mathrm{p}, \pi^{+}\right) \mathrm{X}$ reaction proceeds predominantly through $\mathrm{p}$-p collisions, it was expected that the higher proton/neutron ratio in water than in copper would result in an enhanced $\pi^{+}$, and hence neutrino, production. Detailed Monte Carlo studies[3] showed an increase in the LAMPF $\nu$ flux of $24 \%$; since the high-Z ISIS spallation targets have a yet lower proton/neutron ratio, one would expect a much greater proportional neutrino flux increase for ISIS.

Water targets for insertion into the ISIS target were modelled as simple steel cylindrical shells filled with $\mathrm{D}_{2} \mathrm{O}$, with a $0.4 \mathrm{~cm}$ Inconel disk in the center to represent the internal baffles neccessary for efficient water flow and heat extraction. Water-cooled windows at both ends were modelled as two $5 \mathrm{~cm}$ diameter flat Inconel disks separated by $\mathrm{D}_{2} \mathrm{O}$. Water targets were placed in either of two positions relative to the spallation target: referring to Fig. 3, either (1) close to the spallation target, $0.3 \mathrm{~cm}$ from the first spallation plate, or (2) upstream of the ISIS target entry window, $25.0 \mathrm{~cm}$ from the first 
spallation plate.

Table 3: Calculations with 10 and $20 \mathrm{~cm} \mathrm{D}_{2} \mathrm{O}$ targets at two distances from either tantalum or uranium spallation targets. A proton beam energy of $800 \mathrm{MeV}$ was used. The absolute neutrino flux is given in column 4, and the fractional flux increase in column 5 .

\begin{tabular}{lcccc}
\hline $\begin{array}{l}\text { Spallation } \\
\text { Target }\end{array}$ & $\begin{array}{c}\mathrm{D}_{2} \mathrm{O} \\
\text { Target } \\
(\mathrm{cm})\end{array}$ & $\begin{array}{c}\text { Distance } \\
\text { To ISIS } \\
(\mathrm{cm})\end{array}$ & $\begin{array}{c}\text { Neutrino } \\
\text { Flux } \\
\left(\nu \mathrm{p}^{-1}\right)\end{array}$ & $\begin{array}{c}\text { Neutrino } \\
\text { Increase } \\
(\text { Fraction })\end{array}$ \\
\hline ISIS-Ta & 20 & 25.0 & 0.0743 & 1.66 \\
ISIS-Ta & 20 & 0.3 & 0.0750 & 1.67 \\
ISIS-Ta & 10 & 0.3 & 0.0623 & 1.39 \\
ISIS-U & 20 & 0.3 & 0.0721 & 1.80 \\
\hline
\end{tabular}

Calculations were made with the code KARMEN of the stopped $\pi^{+}$rate, and hence the neutrino flux. For a water target close to the tantalum spallation target and an 800 $\mathrm{MeV}$ incident proton beam, the calculations yield the results shown in Fig. 2. Water targets from 0 to $20 \mathrm{~cm}$ were modelled, and the neutrino fluxes normalized to the 0 $\mathrm{cm}$ water target (corresponding to the normal ISIS spallation target). The apparent offset at $0 \mathrm{~cm}$ water length is due to the increased production from the Inconel windows compared to the tantalum. It is seen that the increase in the stopped $\pi^{+}$rate, and hence the $\nu$ flux, is considerable: a $67 \%$ increase in the $\nu$ flux for a $20 \mathrm{~cm}$ long water target. Further, the $\nu$ flux increase is approximately linear, so estimating the flux increase for a particular target length is straightforward.

Calculations, at $800 \mathrm{MeV}$ incident proton energy, of the $\nu$ flux for several combinations of spallation target material, water target thickness, and water target position are listed in Table 3. The ISIS spallation target is either tantalum (ISIS-Ta) or uranium (ISIS-U). The fractional flux increase is based upon comparison with the $\nu$ fluxes in Table 1. As shown in the calculations for the $20 \mathrm{~cm} \mathrm{D}_{2} \mathrm{O}$ target, in conjunction with the tantalum spallation target, there is little effect upon the neutrino flux of the two water target positions investigated. There is a small difference between the tantalum and the uranium target flux that is consistent with the proportion of $\pi^{+}$that is produced in the spallation targets compared to that produced in the $\mathrm{D}_{2} \mathrm{O}$ targets.

\section{Neutron fluxes for added heavy-water targets}

Since the spallation neutron source at ISIS is used mainly for neutron scattering studies of materials, it is important to estimate how the addition of a neutrino production target will affect the largest group of ISIS users. 


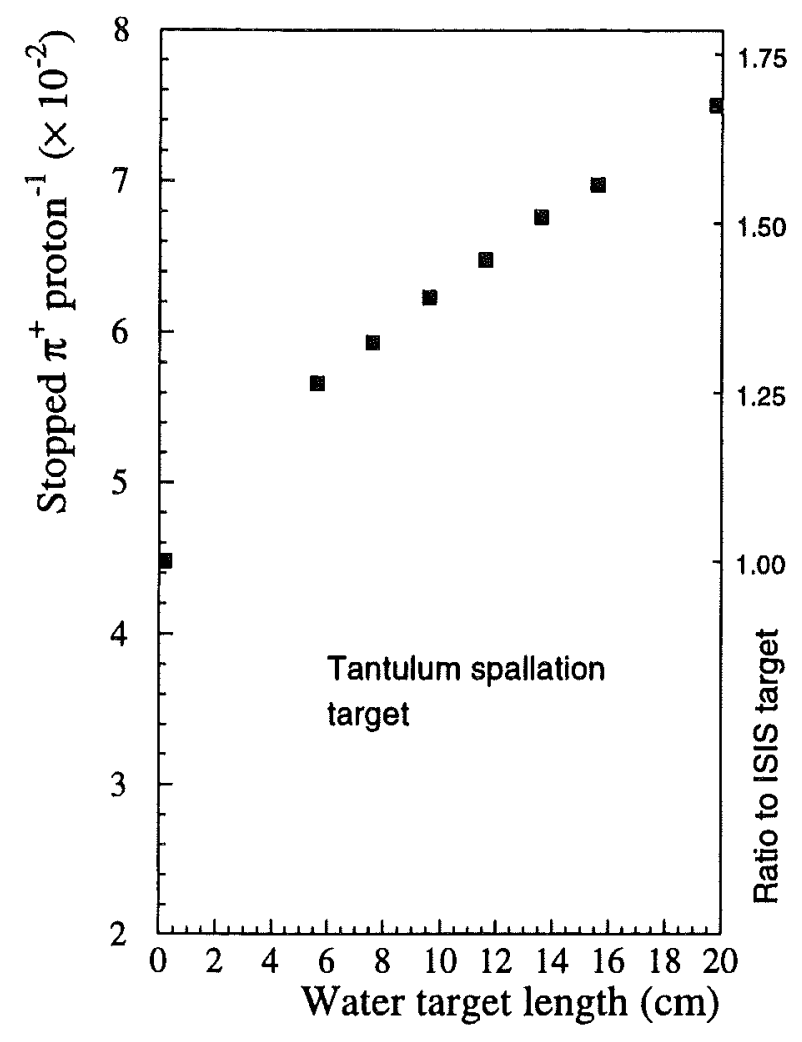

Figure 2: Neutrino fluxes for varying thicknesses of a $\mathrm{D}_{2} \mathrm{O}$ target placed 0.3 $\mathrm{cm}$ upstream of the tantalum spallation target. The ISIS proton beam is $800 \mathrm{MeV}$. The apparent offset at $0 \mathrm{~cm}$ water length is due to increased production from Inconel windows compared to tantalum.

We used a computer model of the ISIS target station to calculate the impact of the neutrino production target on the neutron beamline intensities. The computer model was developed recently for use with the LAHET Code System (LCS) - a family of radiation transport codes developed at the Los Alamos National Laboratory and the Oak Ridge National Laboratory, and used worldwide for the design and analysis of nuclear systems [6]. The computer model was developed to perform a detailed neutronic study of the ISIS target station. The results of this study are described elsewhere [7]. The computer model has many of the essential engineering features of the 'as-built' target station at ISIS. When the computer model was constructed, details that were thought to be important from a neutronics or thermal hydraulics standpoint were included. The LCS computer model is shown in Fig. 3.

As mentioned above, ISIS operates with either a tantalum target (ISIS-Ta), or with a depleted uranium target (ISIS-U). The target consists of a sequence of 23 disks of variable thicknesses contained in a stainless steel canister and is cooled with $\mathrm{D}_{2} \mathrm{O}$. The neutrons 


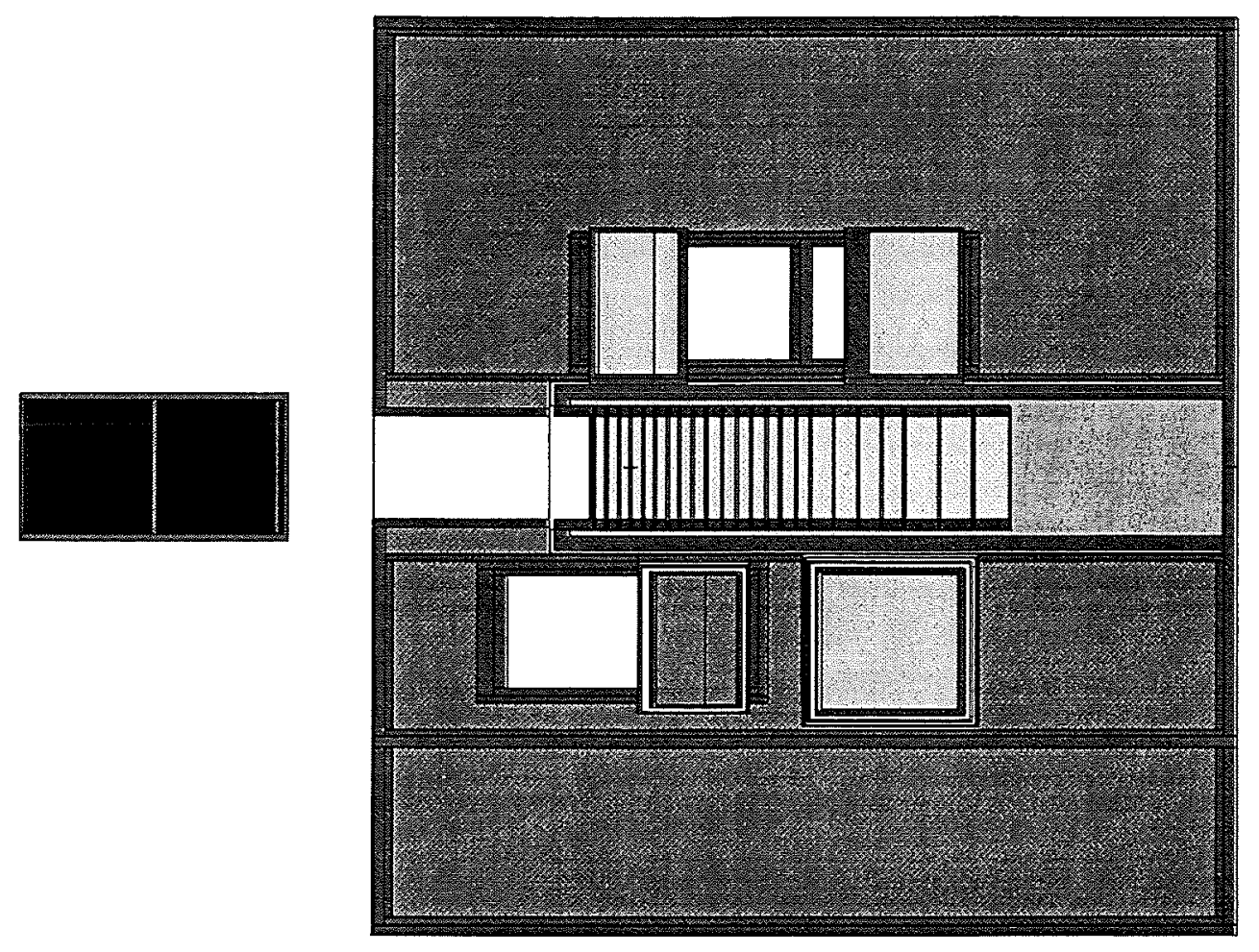

Figure 3: LCS computer model of the ISIS spallation target assembly with the proton beam incident from the left. The two light-water moderators are above the spallation target, the two cryogenic moderators are below. A $20 \mathrm{~cm}$ water target is shown, placed upstream of the spallation target by $25.0 \mathrm{~cm}$.

produced by spallation in the target are too energetic for materials studies: they have to be slowed down to thermal energies before they can be used in neutron scattering experiments. The target is therefore surrounded by four "moderators". The moderating medium used at spallation neutron sources is typically a hydrogen-rich liquid. As shown in Fig.3, ISIS has four moderators: two light water moderators, one liquid methane moderator, and one liquid hydrogen moderator. The water moderators are placed sideby-side above the neutron production target, whereas the cryogenic moderators are located side-by-side underneath the target. The moderating medium is contained in small rectangular aluminum canisters. Each "upstream" moderator (water and liquid methane) has two viewed surfaces, from which neutron beams are extracted. Each "downstream" moderator (water and liquid hydrogen) has only one viewed surface. The 
target-moderator assembly is surrounded by a heavy-water-cooled beryllium reflector to increase the neutronic efficiency of the target station $[8,9]$.

The degradation of the proton beam following the insertion of a neutrino production target upstream of the neutron production target has a negative impact on neutron production. Large energy losses due to ionization in the heavy water target not only decrease the average energy of the protons incident on the neutron production target, but also the intense Coulomb scattering causes changes in proton directions such that some protons never reach the neutron production target. While the latter effect is small, affecting only 2 or $3 \%$ of the protons in the beam, the former is much more serious. Indeed, calculations indicate that after traversing $20 \mathrm{~cm}$ of $\mathrm{D}_{2} \mathrm{O}$, the average proton energy has decreased from $800 \mathrm{MeV}$ to approximately $750 \mathrm{MeV}$. Furthermore, roughly $6 \%$ of the protons now have energies distributed between tens of $\mathrm{MeV}$ and $750 \mathrm{MeV}$. This degradation of the proton beam affects neutron production quite dramatically. Spallation neutrons are produced in two steps: intranuclear cascade and nuclear evaporation [6]. During the intranuclear cascade phase, neutrons and protons are directly ejected from the target nucleus by the incident proton. The nucleons thus produced have typically very high energies, ranging from 10 to $800 \mathrm{MeV}$. Because of their high energy, it is virtually impossible to moderate these intranuclear cascade neutrons. The intranuclear cascade leaves the target nucleus in a highly excited state. During the evaporation phase, the nucleus relaxes to its ground state by emitting low energy (a few $\mathrm{MeV}$ ) neutrons. These neutrons represent the bulk (approximately 80 to $90 \%$ ) of the spallation neutron production, and can be moderated much more easily.

Table 4: Thermal neutron intensities at ISIS-Ta and ISIS-U for a $20 \mathrm{~cm}$ water target located directly in front of the neutron production target. The numbers are intensities relative to the corresponding intensities without a water target.

\begin{tabular}{lcc}
\hline Moderator & ISIS-Ta & ISIS-U \\
\hline Upstream water "POLARIS side" & 0.72 & 0.94 \\
Upstream water "HET side" & 0.73 & 0.93 \\
Upstream liquid methane "HRPD side" & 0.75 & 0.92 \\
Upstream liquid methane "SANDALS side" & 0.79 & 0.93 \\
Downstream water & 0.68 & 0.89 \\
Downstream liquid hydrogen & 0.69 & 0.89 \\
\hline
\end{tabular}

For ISIS-Ta, introducing $20 \mathrm{~cm}$ of water in the proton beam reduces the total spallation neutron production from 16.1 neutrons per incident proton to $11.7 \mathrm{n} / \mathrm{p}$, i.e., a $30 \%$ reduction in neutron production. To this production we must add approximately $1 \mathrm{n} / \mathrm{p}$ from $(n, x n)$ reactions (net production). The $(n, x n)$ production does not seem to depend very much on whether the water target is present.

For ISIS-U, the introduction of $20 \mathrm{~cm}$ of water in the proton beam reduces the spal- 
lation neutron production from $18.9 \mathrm{n} / \mathrm{p}$ to $12.9 \mathrm{n} / \mathrm{p}$, which is also a $30 \%$ reduction. In a depleted uranium target, however, a significant contribution to total neutron production originates from neutron-induced fission, as well as $(n, x n)$ reactions. The contribution from fission and $(\mathrm{n}, \mathrm{xn})$ reactions is of the order of $5 \mathrm{n} / \mathrm{p}$ (net production), and does not seem to depend a great deal on whether the water target is present or not. Notice that the neutrons born in fission and ( $n, x n)$ reactions have energies of a few $\mathrm{MeV}$, and are easily moderated.

To summarize, in a tantalum target, a $30 \%$ reduction in spallation neutron production translates into a $30 \%$ reduction in neutrons that can be moderated, i.e., the evaporation neutrons. The $(\mathrm{n}, \mathrm{xn})$ neutron production is only a small fraction of the neutrons that can be moderated. In the case of a depleted uranium target, we also observe a $30 \%$ reduction in spallation neutron production, but this reduction does not translate into a $30 \%$ reduction in the number of easily-moderated neutrons because the fission and $(n, x n)$ reactions production is a very significant fraction of the total production. In view of these results, it would seem reasonable to conclude that the production of thermal neutrons at ISIS-Ta would be more dramatically affected by the presence of a water target than ISIS-U. This is indeed what our calculations indicate.

Table 5: Thermal neutron intensities at ISIS-Ta for two locations of a $20 \mathrm{~cm}$ water target. The numbers are intensities relative to the corresponding intensities without a water target.

\begin{tabular}{lcc}
\hline Moderator & $\begin{array}{c}\text { ISIS-Ta } \\
\text { @0.3cm }\end{array}$ & $\begin{array}{c}\text { ISIS-Ta } \\
\text { @25cm }\end{array}$ \\
\hline Upstream water "POLARIS side" & 0.72 & 0.65 \\
Upstream water "HET side" & 0.73 & 0.64 \\
Upstream liquid methane "HRPD side" & 0.75 & 0.64 \\
Upstream liquid methane "SANDALS side" & 0.79 & 0.66 \\
Downstream water & 0.68 & 0.60 \\
Downstream liquid hydrogen & 0.69 & 0.62 \\
\hline
\end{tabular}

In order to determine the impact of the neutrino production target on thermal neutron production, we adopted a somewhat arbitrary figure of merit, namely the integrated neutron flux from 0 to $100 \mathrm{meV}$ (arguably the range of neutron energies of interest for neutron scattering studies of materials). Table 4 shows the reduction in thermal beam intensity due to a $20 \mathrm{~cm}$ water target placed directly in front of the neutron production target. As explained above, the reduction is more severe for ISIS-Ta. It is also apparent from Table 4 that the decrease in thermal neutron intensity is slightly greater for the downstream moderators.

The effect of water target location is illustrated in Table 5 for ISIS-Ta. The two water target locations considered are $0.3 \mathrm{~cm}$ and $25 \mathrm{~cm}$ in front of the neutron production 
target, as explained in Section 3. Again, the downstream moderators are somewhat more affected than the upstream moderators. The decrease in thermal neutron beam intensity is larger when the target is located farther away $(25 \mathrm{~cm})$ from the neutron production target. This is very likely due to the fact that the scattering of protons from the beam is felt more acutely when the water target is farther away; simple geometry considerations show that more (scattered) protons miss the neutron production target as the distance between the two targets increases. In addition, the presence of $20 \mathrm{~cm}$ of heavy water directly in front of the neutron production target has a neutron reflection effect that probably helps somewhat in reducing the decrease in thermal neutron beam intensity when the water target is only $0.3 \mathrm{~cm}$ away from the neutron production target.

Table 6: Thermal neutron intensities at ISIS-Ta for two water target lengths, 10 and 20 $\mathrm{cm}$. The water target is located $0.3 \mathrm{~cm}$ in front of the neutron production target. The numbers are intensities relative to the corresponding intensities without a water target.

\begin{tabular}{|c|c|c|}
\hline Moderator & $\begin{array}{c}\text { ISIS-Ta } \\
20 \mathrm{~cm} \mathrm{D} \mathrm{D}_{2} \mathrm{O} \text { target }\end{array}$ & $\begin{array}{c}\text { ISIS-Ta } \\
10 \mathrm{~cm} D_{2} O \text { target }\end{array}$ \\
\hline Upstream water "POLARIS side" & 0.72 & 0.84 \\
\hline Upstream water "HET side" & 0.73 & 0.85 \\
\hline Upstream liquid methane "HRPD side" & 0.75 & 0.85 \\
\hline Upstream liquid methane "SANDALS side" & 0.79 & 0.87 \\
\hline Downstream water & 0.68 & 0.80 \\
\hline Downstream liquid hydrogen & 0.69 & 0.82 \\
\hline
\end{tabular}

Since ISIS runs quite frequently with a tantalum target, and given that the losses in thermal neutron beam intensities incurred from a $20 \mathrm{~cm}$ water target are not acceptable, we repeated our calculations for a $10 \mathrm{~cm}$ water target, $0.3 \mathrm{~cm}$ in front of the neutron production target. The results are summarized in Table 6.

The losses with a $10 \mathrm{~cm}$ long neutrino production target are still significant. One could further reduce the losses by going to a "tighter" proton beam focus. Table 7 compares the reduction in intensity for a typical ISIS production-proton beam (Gaussian beam profile, $\sigma=1.5 \mathrm{~cm}$ ) and a more tightly focussed beam (parabolic beam profile, $2.5 \mathrm{~cm}$ diameter). A tighter focus helps somewhat, at least for the upstream moderators, but the effect is essentially nil on the downstream moderators.

\section{$5 \quad$ Neutrino and neutron flux comparisons}

Comparisons of the calculated neutron and neutrino fluxes for different combinations of spallation target material, and $\mathrm{D}_{2} \mathrm{O}$ target length and position, are displayed in Table 8. The first two entries show that while the placement of the $\mathrm{D}_{2} \mathrm{O}$ target relative to the spallation target has little effect upon the neutrino flux, it can change greatly the 
Table 7: Thermal neutron intensities at ISIS-Ta for a $10 \mathrm{~cm}$ water target length and two proton beam profiles. The water target is located $0.3 \mathrm{~cm}$ in front of the neutron production target. The numbers are intensities relative to the corresponding intensities without a water target.

\begin{tabular}{lcc}
\hline Moderator & $\begin{array}{c}\text { ISIS-Ta } \\
\text { Gaussian beam }\end{array}$ & $\begin{array}{c}\text { ISIS-Ta } \\
\text { Parabolic beam }\end{array}$ \\
\hline Upstream water "POLARIS side" & 0.84 & 0.89 \\
Upstream water "HET side" & 0.85 & 0.86 \\
Upstream liquid methane "HRPD side" & 0.85 & 0.90 \\
Upstream liquid methane "SANDALS side" & 0.87 & 0.90 \\
Downstream water & 0.80 & 0.80 \\
Downstream liquid hydrogen & 0.82 & 0.82 \\
\hline
\end{tabular}

neutron flux. For the $\mathrm{D}_{2} \mathrm{O}$ target $24.8 \mathrm{~cm}$ back from the spallation target, the neutron flux reduction worsens from $26 \%$ to $37 \%$. As the third entry shows, a half-sized $\mathrm{D}_{2} \mathrm{O}$ target produces both half the neutrino increase and half the neutron decrease.

The fourth entry is for a uranium spallation target, with a $20 \mathrm{~cm} \mathrm{D}_{2} \mathrm{O}$ target close to the first plate of the spallation target. It is seen that the neutrino production is again sharply increased, this time by $80 \%$. The neutron flux, in contrast, is reduced only by about $8 \%$. As explained in Section 4 , the much smaller effect of the $\mathrm{D}_{2} \mathrm{O}$ target upon neutron flux is due to the fact that fission and (,$x n)$ neutron production is a large fraction of the total neutron production, and this fraction is virtually independent of the presence of the neutrino production target. Since these low-energy (a few MeV) neutrons couple much better to the beam-line moderators, there is only a relatively small $6-10 \%$ decrease in neutron fluxes. It should particularly be noted that a uranium target, for the same reasons, leads to almost twice the absolute neutron fluxes than a tantalum target.

There is a large increase in the low- $Z$ material seen by decaying $\pi^{-}$and $\mu^{-}$, when the $\mathrm{D}_{2} \mathrm{O}$ target is added to the facility, resulting in a significant increase in the $\bar{\nu}_{e}$ background. This produces the doubling of the ratio of $\bar{\nu}_{e}$ to $\nu_{e}$, in the last column of Table 8 compared to the last column of Table 1 . However, the time structure in the ISIS proton beam enables this background to be substantially reduced. The time spectrum of $\bar{\nu}_{e}$ from the decay at rest of $\mu^{-}$in the heavy water plus uranium spallation target combination (the ISIS-U entry in Table 8) is shown in Fig. 4. Here, the double-peaked initial shape is a consequence of the proton time structure, made up of two $100 \mathrm{~ns}$ wide pulses separated in time by $330 \mathrm{~ns}$, with a repetition rate of $50 \mathrm{~Hz}$. The total $\bar{\nu}_{e}$ time spectrum then consists of two basic components: (1) an exponential decay in low-Z materials $\mathrm{Be}$ and $\mathrm{D}_{2} \mathrm{O}$ dominated by the $2.2 \mu$ s muon mean life, and (2) the much faster exponential decay 
Table 8: Comparison of calculations of neutron and neutrino fluxes for a proton beam energy of $800 \mathrm{MeV}$, and several spallation target and $\mathrm{D}_{2} \mathrm{O}$ target combinations. The range of reduction in neutron fluxes for the different neutron beam lines is shown in column 4.

\begin{tabular}{lccccc}
\hline $\begin{array}{l}\text { Spallation } \\
\text { Target }\end{array}$ & $\begin{array}{c}\mathrm{D}_{2} \mathrm{O} \\
\text { Target } \\
(\mathrm{cm})\end{array}$ & $\begin{array}{c}\text { Distance } \\
\text { To ISIS } \\
(\mathrm{cm})\end{array}$ & $\begin{array}{c}\text { Neutron } \\
\text { Reduction } \\
(\%)\end{array}$ & $\begin{array}{c}\text { Neutrino } \\
\text { Increase } \\
(\text { Fraction })\end{array}$ & $\begin{array}{c}\bar{\nu}_{e} / \nu_{e} \\
\text { Ratio } \\
\left(10^{-3}\right)\end{array}$ \\
\hline ISIS-Ta & 20 & 24.8 & $35-40$ & 1.66 & 1.62 \\
ISIS-Ta & 20 & 0.3 & $21-31$ & 1.67 & 1.13 \\
ISIS-Ta & 10 & 0.3 & $13-20$ & 1.39 & 0.91 \\
ISIS-U & 20 & 0.3 & $6-11$ & 1.80 & 1.13 \\
\hline
\end{tabular}

in high- $\mathrm{Z}$ materials $\mathrm{Fe}, \mathrm{U}$ and $\mathrm{Cu}$ characterized by a large absorption rate.

Because the physics of the KARMEN experiment is normally separated into that due to $\nu_{\mu}$ interactions (from the $26 \mathrm{~ns} \pi^{+}$decay at rest), and that due to $\nu_{e}$ and $\bar{\nu}_{\mu}$ interactions (from the $2.2 \mu \mathrm{s} \mu^{+}$decay at rest), events are separated into two time groups, above and below $600 \mathrm{~ns}$ from the start of the proton pulses. Inspection of Fig. 4 shows that $57 \%$ of the $\bar{\nu}_{e}$ neutrinos are emitted after $600 \mathrm{~ns}$. Thus, the expected experimental background for the ISIS-U plus $\mathrm{D}_{2} \mathrm{O}$ target is reduced from $1.13 \times 10^{-3}$ in the last entry in Table 4 to a value $\bar{\nu}_{e} / \nu_{e}=0.64 \times 10^{-3}$.

\section{Conclusions}

Calculations of the neutrino and neutron fluxes for different configurations of the ISIS spallation neutron source have been presented. With the addition of a $20 \mathrm{~cm}$ (10 cm) $\mathrm{D}_{2} \mathrm{O}$ target immediately upstream of a tantalum spallation source, the neutrino flux is increased by $67 \%(39 \%)$ and the neutron flux is decreased by about $25 \%(16 \%)$. While the neutron flux loss for the $20 \mathrm{~cm} \mathrm{D}_{2} \mathrm{O}$ target is probably unacceptably large, the $10 \mathrm{~cm} \mathrm{D}_{2} \mathrm{O}$ target coupled with the tantalum spallation source represents a possible compromise. For this case, the neutrino flux gain of $39 \%$ is still a very useful increase.

The calculated neutron flux reduction was much less for the uranium spallation source and a $20 \mathrm{~cm} \mathrm{D}_{2} \mathrm{O}$ target. As discussed above, for a depleted uranium target, the number of easily-moderated neutrons arising from the larger fission and $(n, x n)$ reactions already makeup most of the useable neutron flux and are not affected by the water target. As calculated, the neutrino flux increased by $80 \%$ (61\% relative to the tantalum spallation target flux) and the neutron flux decreased by $6-11 \%$. Since the neutron flux for a uranium target is twice that for a tantalum target, the use of a depleted uranium target would actually result in a considerable increase in the neutron fluxes available at ISIS. 


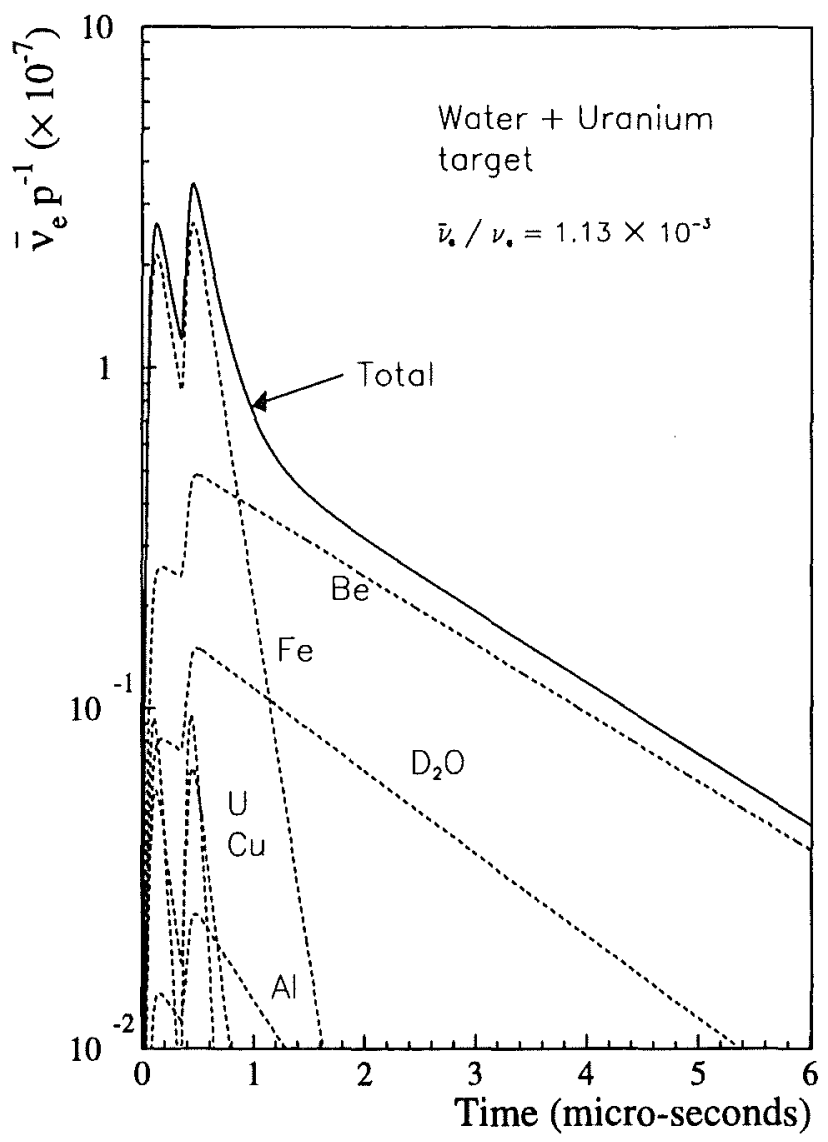

Figure 4: The time spectrum of $\bar{\nu}_{e}$ from the decay-at-rest of $\mu^{-}$in the heavy water plus uranium spallation target combination. The doublepeaked initial shape is due to the proton time structure, while the total $\bar{\nu}_{e}$ time spectrum consists of two basic components, a relatively slow decay rate in low- $\mathrm{Z}$ materials $\left(\mathrm{Be}\right.$ and $\mathrm{D}_{2} \mathrm{O}$ ) and the faster decay rate in high- $\mathrm{Z}$ materials ( $\mathrm{Fe}, \mathrm{U}$ and $\mathrm{Cu}$ ).

This very attractive option would result in roughly $80 \%$ increases in both the neutrino and neutron fluxes. It is, however, technically more challenging, as the handling of depleted uranium under intense radioactivity has many inherent difficulties. The experimental programs in both neutron scattering (materials science) and neutrino interactions (astro/particle physics) are entering extremely exciting periods, and both programs could benefit enormously from increased beam intensities.

We gratefully acknowledge financial support from the Department of Energy (USA) and from the Forschungszentrum Karlsruhe (Germany). We also thank Dr. T. Broome for several useful discussions about the ISIS spallation target facility. 


\section{References}

[1] B. Armbruster, et al., Nucl. Phys. B (Proc. Suppl.) 38 (1995) 198; B. Armbruster, et al., Nucl. Phys. B (Proc. Suppl.) 38 (1995) 235.

[2] T.A. Gabriel, et al., Kernforschungszentrum Karlsruhe report, KfK 3174 (1981).

[3] R.L. Burman, M.E. Potter and E.S. Smith, Nucl. Instr. and Methods A291 (1990) 621.

[4] R.C. Allen, et al., Nucl. Instr. and Methods A284 (1989) 347.

[5] R.L. Burman, A.C. Dodd and P. Plischke, Forschungszentrum Karlsruhe report, FZKA 5595 (1995); to be submitted to Nucl. Instr. and Methods.

[6] R.E. Prael and H. Lichtenstein, "User guide to LCS: The LAHET Code System," Los Alamos National Laboratory report LA-UR-89-3014 (September 1989); "MCNP - A general Monte-Carlo code for neutron and photon transport," Judith F. Briesmeister, editor, Los Alamos National Laboratory report LA-7396-M, Rev.2 (September 1986).

[7] L.L. Daemen, T.A. Broome, and M. Holding, "Neutronic study of the ISIS target station," preprint.

[8] D.A. Gray, "Status of the SNS," in Proceedings of the Eighth Meeting of the International Collaboration on Advanced Neutron Sources, Rutherford-Appleton Laboratory (July 1985), RAL-85-110, Vol.1, G. Sterling, Ed., p.65.

[9] F. Atchison, Rutherford Laboratory report, RL-81-006 (April 1981). 